\title{
A note on mean ergodic composition operators on spaces of holomorphic functions
}

\author{
José Bonet · Pawel Domański
}

Received: 9 March 2010 / Accepted: 7 April 2010 / Published online: 1 February 2011

(C) The Author(s) 2011. This article is published with open access at Springerlink.com

\begin{abstract}
We characterize those composition operators defined on spaces of holomorphic functions of several variables which are power bounded, i.e. the orbits of all the elements are bounded. This condition is equivalent to the composition operator being mean ergodic. We also describe the form of the symbol when the composition operator is mean ergodic.
\end{abstract}

Keywords Composition operators - Mean ergodic operators - Power bounded operators · Spaces of holomorphic functions

Mathematics Subject Classification (2000) $\quad 47 \mathrm{~B} 33 \cdot 46 \mathrm{E} 10 \cdot 46 \mathrm{~A} 04$

\section{Introduction and notation}

The purpose of this note was to study the behaviour of orbits of composition operators $C_{\varphi}(f):=f \circ \varphi, \varphi$ a holomorphic self map, on spaces $H(U)$ of holomorphic functions defined on an open connected subset (=domain) $U$ of $\mathbb{C}^{d}$ or, more generally, of a Stein manifold $\Omega$. The space $H(U)$ is endowed with the compact open topology and it is a nuclear Fréchet space, hence Montel. We are interested here in the case when the orbits of all the

The research of Bonet was partially supported by MEC and FEDER Projects MTM2007-62643 and MTM2010-15200 and by GV Project Prometeo/2008/101. The research of Domański was supported in years 2007-2010 by Ministry of Science and Higher Education, Poland, grant no. NN201 274033.

\footnotetext{
J. Bonet $(\bowtie)$

Inst. Univ. Matemática Pura y Aplicada (IUMPA),

Edificio ID15 (8E), Cubo F, Cuarta Planta,

Universidad Politécnica de Valencia, 46071 Valencia, Spain

e-mail: jbonet@mat.upv.es

P. Domański

Faculty of Mathematics and Computer Science, A. Mickiewicz University Poznań, Umultowska 87, 61-614 Poznań, Poland

e-mail: domanski@amu.edu.pl
} 
elements under $C_{\varphi}$ are bounded. If this occurs, the operator $C_{\varphi}$ is called power bounded. A related interesting question is when $C_{\varphi}$ is mean ergodic $[3,9,17,22]$. We complement important results on universality which were obtained in $[5,6,12]$. These papers do not discuss when $C_{\varphi}$ is power bounded or mean ergodic on $H(U)$.

There is a huge literature about the dynamical behavior of various linear continuous operators on Banach, Fréchet and more general locally convex spaces; see the survey papers by Grosse-Erdmann [10,11] and the recent books by Bayart and Matheron [4] and by GrosseErdmann and Peris [13]. For the general theory of composition operators on Banach spaces of holomorphic functions see [8,20], and for composition operators on $H(U)$ see [21].

Let $T: E \rightarrow E$ be a continuous linear operator on a Hausdorff locally convex space $E$. The iterates of $T$ are denoted by $T^{n}:=T \circ \cdots \circ T, n \in \mathbb{N}$. If the sequence $\left(T^{n}\right)_{n \in \mathbb{N}}$ is equicontinuous in the space $L(E)$ of linear operators from $E$ to $E, T$ is called power bounded. In case $E=H(U)$, it is a Fréchet space and the uniform boundedness principle can be applied to conclude that $T$ is power bounded if and only if the orbit $\left\{T^{n}(x): n \in \mathbb{N}\right\}$ is bounded for every $x \in E$.

A continuous linear operator $T$ on $E$ is called mean ergodic if the limits

$$
P x:=\lim _{N \rightarrow \infty} \frac{1}{N} \sum_{n=1}^{N} T^{n} x, \quad x \in E,
$$

exist in $E$. A power bounded operator $T$ is mean ergodic precisely when $X=\operatorname{ker}(I-T) \oplus$ $\overline{\operatorname{im}(I-T)}$. Moreover, $\operatorname{im} P=\operatorname{ker}(I-T)$ and $\operatorname{ker} P=\overline{\operatorname{im}(I-T)}$. If the space $E$ is barrelled and $T$ is mean ergodic, the sequence $\frac{1}{N} \sum_{n=1}^{N} T^{n}$ converges pointwisely to a continuous linear projection $P$; see [22, Ch. VIII, Sect. 3]. If the convergence is uniform on bounded sets we call $T$ uniformly mean ergodic. There is a classical theory of mean ergodic operators which goes back to fundamental papers of Yosida and Hille especially in the Banach case; cf. [17]. For more details on the locally convex theory see $[2,3,22]$ and the references therein.

We characterize those composition operators $C_{\varphi}, C_{\varphi}(f):=f \circ \varphi$, on the space of holomorphic functions $H(U)$ on a domain $\mathrm{U}$ in a Stein manifold $\Omega, \varphi: U \rightarrow U$ holomorphic, such that $C_{\varphi}$ is power bounded; see Proposition 1 . We prove that power boundedness is equivalent to (uniform) mean ergodicity. Finally in Theorem 1 we describe precisely the form of $\varphi$ such that $C_{\varphi}$ is power bounded. The evaluation of these equivalent conditions and the precise form of the projection which appears as limit of the Cesaro sums is determined in concrete cases in Corollaries 2 and 3. Our results here are utilized in [7] to investigate power bounded composition operators on spaces of real analytic functions.

We will use Kobayashi semi-distance $k_{V}(\cdot, \cdot)$ on a complex manifold $V \subseteq \mathbb{C}^{d}$. Here the beautiful book [16] is a standard reference. Every holomorphic map $\varphi: V \rightarrow V$ is always non-expansive with respect to $k_{V}$. The manifold $V$ is called hyperbolic if $k_{V}$ is a distance (and then it induces the standard topology of $V$ ). Every domain biholomorphic to a bounded set is automatically hyperbolic [16, Cor. 4.1.10, Prop. 3.2.2]. The manifold $V$ is called Kobayashi complete if $\left(V, k_{V}\right)$ is a complete metric space, or equivalently, if every ball in this space is relatively compact [16, Prop. 1.1.9]. Every bounded open set $V \subseteq \mathbb{C}^{d}$ such that every boundary point admits a weak peak function is Kobayashi complete [16, Cor. 4.1.11]. Both hyperbolicity and Kobayashi completeness are biholomorphic invariants. Recall that every Stein manifold embeds via a proper map into $\mathbb{C}^{d}$ for suitable $d \in \mathbb{N}[15,5.3 .9]$.

By $B(x, r)$ and $B_{k_{V}}(x, r)$ we denote, respectively, Euclidean and Kobayashi balls of center $x$ and radius $r$. The notation $K \Subset U$ means that $K$ is a compact subset of the open set $U$. For non-explained notions from functional analysis we refer to [18]. For complex analysis of several variables see [14]. For dynamics of holomorphic maps see [1,19]. 


\section{Power bounded composition operators on spaces of holomorphic functions}

Let $\varphi: U \rightarrow U$ be a continuous self-map on a topological space $U$, we say that $\varphi$ has stable orbits on $U$ if for every compact subset $K$ of $U$ there is a compact subset $L \Subset U$ such that $\varphi^{n}(K) \subseteq L$ for $n \in \mathbb{N}$. This concept plays an important role later on.

Proposition 1 Let $U$ be a connected domain of holomorphy in $\mathbb{C}^{d}$ (or even in a Stein manifold) and let $\varphi: U \rightarrow U$ be a holomorphic mapping. The following assertions are equivalent:

(a) $C_{\varphi}: H(U) \rightarrow H(U)$ is power bounded.

(b) $C_{\varphi}: H(U) \rightarrow H(U)$ is uniformly mean ergodic.

(c) $C_{\varphi}: H(U) \rightarrow H(U)$ is mean ergodic.

(d) The map $\varphi$ has stable orbits on $U$.

(e) There is a fundamental family of connected compact sets $\left(L_{j}\right)$ in $U$ such that $\varphi\left(L_{j}\right) \subseteq$ $L_{j}$ for every $j \in \mathbb{N}$.

Proof $(\mathrm{a}) \Rightarrow(\mathrm{b})$ : Follows from [2, Prop. 2.4] since $H(U)$ is a Fréchet Montel space.

(b) $\Rightarrow$ (c): Obvious.

(c) $\Rightarrow\left(\right.$ d): By [2, Prop. 2.2], the sequence $\left(\frac{1}{n} C_{\varphi^{n}}\right)$ tends to zero, so it is bounded and equicontinuous on $H(U)$. For every $K$ compact in $U$ we find $L$ compact in $U$ such that $(1 / n) C_{\varphi^{n}}$ extends to $(1 / n) \widehat{C_{\varphi^{n}}}: A(L) \rightarrow A(K)$ as a continuous map, where $K$ and $L$ have non-empty interior and $A(K)$ denotes the completion of $H(U)$ with respect to the sup-norm on $K$. Since $U$ is a domain of holomorphy it is holomorphically convex and we can assume that $L$ is equal to its $U$-holomorphic hull, i.e.,

$$
L=\left\{w \in U:|f(w)| \leq \sup _{z \in L}|f(z)| \forall f \in H(U)\right\} .
$$

Suppose that there are $n \in \mathbb{N}$ and $a \in \varphi^{n}(K) \backslash L$. Then there is a function $f \in H(U)$ such that $f(a)=1$ but $\sup _{z \in L}|f(z)| \leq \varepsilon, \varepsilon \in(0,1)$. Since $f^{k}$ tends uniformly to zero on $L$ as $k \rightarrow \infty$ but does not tend to zero at $a$, the map $C_{\varphi^{n}}$ is not continuous from $A(L)$ to $A(K)$; a contradiction.

(d) $\Rightarrow$ (e): Let $K$ be connected compact set in $U$. Select a connected compact set $L \supseteq K$ such that $\varphi^{n}(K) \subseteq L$ for $n \in \mathbb{N}$. Apply condition (d) again to find a compact set $M$ such that $\varphi^{n}(L) \subseteq M$ for every $n \in \mathbb{N}$. Then

$$
\tilde{L}:=\overline{L \cup \bigcup_{n=1}^{\infty} \varphi^{n}(L)}
$$

is a compact connected set such that $\varphi(\tilde{L}) \subseteq \tilde{L}$ and $\tilde{L} \supseteq K$. It is connected since $\varphi^{n}(L)$ is connected and $\varphi^{n}(L) \cap L$ contains $\varphi^{n}(K)$. By induction we can construct $\left(L_{j}\right)$ as required. (e) $\Rightarrow($ a): Obvious.

Remark 1. The conditions (d) and (e) in Proposition 1 are always equivalent. The implication (c) $\Rightarrow$ (d) requires in the proof that $U$ is a domain of holomorphy, the converse implication does not-it suffices that $U$ is a domain in a Stein manifold.

Remark 2. Every domain $U$ in a Stein manifold has a unique maximal extension $V$ such that $V$ is a Stein manifold and $H(U)$ and $H(V)$ are isomorphic as Fréchet algebras (points of $V$ are exactly multiplicative functionals on $H(U)$, see [14, Sect. I.H, Th. III.J.1, III.R.5]). Since $C_{\varphi}: H(U) \rightarrow H(U)$ is an algebra homomorphism, $\varphi$ extends uniquely to $\psi: V \rightarrow V$ 
such that $C_{\varphi}$ corresponds to $C_{\psi}$. These remarks permit us to conclude that the equivalence (a) $\Leftrightarrow(\mathrm{b}) \Leftrightarrow$ (c) in Proposition 1 holds for arbitrary domain $U$ in a Stein manifold.

We analyze the holomorphic functions $\varphi: U \rightarrow U$ for which condition (d) in Theorem 1 is satisfied.

Proposition 2 Let $\varphi: U \rightarrow U$ be a holomorphic map and let $U$ be a domain in a Stein manifold. Assume that there is a fundamental family of compact subsets $\left(L_{j}\right)$ of $U$ such that $\varphi\left(L_{j}\right) \subseteq L_{j}$ for every $j \in \mathbb{N}$. Then for every $j \in \mathbb{N}$ there is a fundamental family of (open!) neighbourhoods $V$ of $L_{j}$ in $U$ such that $\varphi(V) \subseteq V$. In particular, every compact set $K \subseteq U$ is contained in a hyperbolic open subset $V \subseteq U$ such that $\varphi(V) \subseteq V$.

Proof Clearly $U$ is a manifold embedded into $\mathbb{C}^{d}$. Without loss of generality we may assume that $L_{j} \Subset \stackrel{\circ}{L}_{j+1}$. Fix $j \in \mathbb{N}$. There is $\varepsilon>0$ such that $\left(L_{j}+B(0, \varepsilon)\right) \cap U \subseteq L_{j+1}$. Here $L+B(0, \varepsilon)$ means in fact the algebraic sum of $L$ and $B(0, \varepsilon)$ in $\mathbb{C}^{d}$. By the Cauchy estimates, the sequence $\left(\varphi^{n}\right)_{n}$ is uniformly equicontinuous on $L_{j+2}$. Then

$$
\begin{gathered}
\forall \delta>0 \exists 0<\eta(\delta)<\delta \quad \forall n \in \mathbb{N}: \quad x, y \in L_{j+1}, \\
\|x-y\|<\eta(\delta) \Rightarrow\left\|\varphi^{n}(x)-\varphi^{n}(y)\right\|<\delta .
\end{gathered}
$$

Let us define $W_{0}:=L_{j}, \quad W_{k}:=\left(\varphi\left(W_{k-1}\right)+B\left(0, \eta\left(\frac{\varepsilon}{2^{k}}\right)\right)\right) \cap U$ and prove inductively that

$$
W_{k} \subseteq L_{j+1} \quad \text { and } \quad \varphi^{n}\left(W_{k}\right) \subseteq\left(L_{j}+B\left(0, \sum_{j=1}^{k} \frac{\varepsilon}{2^{j}}\right)\right) \cap U .
$$

The condition is true for $k=0$. Now, assuming (2) for $k-1$ we get

$$
\begin{aligned}
W_{k} & =\left(\varphi\left(W_{k-1}\right)+B\left(0, \eta\left(\frac{\varepsilon}{2^{k}}\right)\right)\right) \cap U \\
& \subseteq\left(L_{j}+B\left(0, \sum_{j=1}^{k-1} \frac{\varepsilon}{2^{j}}\right)+B\left(0, \eta\left(\frac{\varepsilon}{2^{k}}\right)\right)\right) \cap U \subseteq L_{j+1} .
\end{aligned}
$$

Moreover, we have

$$
\begin{aligned}
\varphi^{n}\left(W_{k}\right) & =\varphi^{n}(\underbrace{\left(\varphi\left(W_{k-1}\right)+B\left(0, \eta\left(\frac{\varepsilon}{2^{k}}\right)\right)\right) \cap U}_{\subseteq L_{j+1}}) \subseteq\left(\varphi^{n+1}\left(W_{k-1}\right)+B\left(0, \frac{\varepsilon}{2^{k}}\right)\right) \cap U \\
& \subseteq\left(L_{j}+B\left(0, \sum_{j=1}^{k-1} \frac{\varepsilon}{2^{j}}\right)+B\left(0, \frac{\varepsilon}{2^{k}}\right)\right) \cap U \subseteq\left(L_{j}+B\left(0, \sum_{j=1}^{k} \frac{\varepsilon}{2^{j}}\right)\right) \cap U .
\end{aligned}
$$

Since $W_{k}$ are open subsets of $U$ and $\varphi\left(W_{k}\right) \subseteq W_{k+1}$ thus $\tilde{W}:=\bigcup_{k=0}^{\infty} W_{k}$ is an open set in $U$ such that $\varphi(\tilde{W}) \subseteq \tilde{W} \subseteq L_{j}+B(0, \varepsilon)$. Taking $\varepsilon>0$ arbitrarily small we get the conclusion. Since every submanifold of a bounded open set in $\mathbb{C}^{d}$ is hyperbolic [16, Cor. 4.1.10, Prop. 3.2.2], the last sentence of the statement follows.

Proposition 3 Let $\varphi: U \rightarrow U$ be a holomorphic map and let $U$ be a domain in a Stein manifold. 
(a) If $U$ is hyperbolic and every orbit of $\varphi$ in $U$ is relatively compact then $\varphi$ has stable orbits in $U$ and, hence, $C_{\varphi}: H(U) \rightarrow H(U)$ is power bounded.

(b) If $U$ is Kobayashi complete hyperbolic and there is a relatively compact orbit of $\varphi$ then $\varphi$ has stable orbits in $U$ and, hence, $C_{\varphi}: H(U) \rightarrow H(U)$ is power bounded.

Proof (a) Fix a compact set $K$. For every $x \in K$ its $\varphi$-orbit $O_{x}:=\left\{\varphi^{n}(x): n \in \mathbb{N}\right\}$ is relatively compact. Hence there is $\varepsilon(x)>0$ such that $U_{x}:=\left\{z \in U: k_{U}\left(z, O_{x}\right)<\varepsilon(x)\right\}$ is relatively compact in $U$. As $\left(B_{k_{U}}(x, \varepsilon(x))_{x \in K}\right.$ is a covering of $K$, there is a finite subcovering $\left(B_{k_{U}}\left(x_{i}, \varepsilon\left(x_{i}\right)\right)\right)_{i=1, \ldots, m}$. Since $\varphi$ is non-expansive with respect to the Kobayashi metric of $U$, for each $z \in K, \varphi^{n}(z) \in \bigcup_{i=1}^{m} U_{x_{i}}$ for each $z \in K$, and the set $\bigcup_{i=1}^{m} U_{x_{i}}$ is relatively compact.

(b) Let the $\varphi$-orbit $O_{x}$ of $x$ be relatively compact. Let $K$ be an arbitrary compact subset of $U$ containing $O_{x}$, thus $K \subseteq B_{k_{U}}(x, r)$ for some $r>0$. Since $\varphi$ is non-expansive with respect to the Kobayashi metric of $U$, orbits of all points of $K$ are contained in $B_{k_{U}}(x, 2 r)$. This set is relatively compact since in a complete hyperbolic set all Kobayashi balls are relatively compact.

Remark For $U=\mathbb{C}$, part (b) of Proposition 3 does not hold: it is enough to take $\varphi(z)=2 z$.

Corollary 1 Let $U$ be a topologically contractible bounded strongly pseudoconvex domain in $\mathbb{C}^{d}$ with $\mathcal{C}^{3}$ boundary. Let $\varphi: U \rightarrow U$ be a holomorphic map. Then $C_{\varphi}: H(U) \rightarrow H(U)$ is power bounded if and only if $\varphi$ has a fixed point.

Proof By Proposition 1, if $C_{\varphi}$ is power bounded $\varphi^{n}$ cannot converge to a boundary point. By [16, Th. 5.5.9], if $\varphi^{n}$ does not converge to a boundary point then it has a fixed point. By [16, Cor. 4.1.12], $U$ is complete hyperbolic. Sufficiency follows from Proposition 3 (b).

A precise description of maps $\varphi$ such that $C_{\varphi}: H(U) \rightarrow H(U)$ is power bounded follows from an argument of Abate originally used for taut manifolds, see [16, Th. 5.5.4].

Theorem 1 Let $U$ be a domain in a Stein manifold and let $\varphi: U \rightarrow U$ be a holomorphic map. If $\varphi$ has stable orbits on $U$, then there is a holomorphic submanifold $M$ of $U$ and $a$ holomorphic surjective retraction $\rho: U \rightarrow M$ such that $\psi:=\left.\varphi\right|_{M}$ is an automorphism of M. Moreover,

$$
G:={\overline{\left\{\psi^{n}: n \in \mathbb{N}\right\}}}^{H(M, M)}
$$

is a compact abelian group of automorphisms on $M$ such that every cluster point of $\left(\varphi^{n}\right)$ in $H(U, U)$ is of the form $\gamma \circ \rho$ where $\gamma \in G$. The $\varphi$-orbit of every element $z \in U$ tends to some $G$-orbit of elements of $M$, in particular, it tends to $M$ and this convergence is uniform with respect to $z$ belonging to a fixed compact set.

Finally,

$$
P(f)(z):=\lim _{N \rightarrow \infty} \frac{1}{N} \sum_{n=1}^{N} C_{\varphi^{n}}(f)(z)=\int_{G} f(\gamma \circ \rho(z)) d H(\gamma),
$$

where $H$ is the Haar measure on $G$, and the image of the projection P satisfies

$$
\operatorname{im} P=\left\{f: f \text { is constant on } \rho^{-1}(\{\gamma \circ \rho(z): \gamma \in G\}) \quad \forall z \in U\right\} .
$$


Proof Most of the statement follows immediately form the proof of [16, Th. 5.5.4] except the uniform convergence of orbits and description of the projection $P$. We prove this part below.

Since every sequence $\left(\varphi^{n_{j}}\right)$ contains a subsequence convergent to $\gamma \circ \rho$ for some $\gamma \in G$ it follows that the set of all cluster points of the orbit:

$$
O_{x}:=\left\{\varphi^{n}(x): n \in \mathbb{N}\right\}
$$

is exactly equal to

$$
G_{\rho(x)}:=\{\gamma \circ \rho(x): \gamma \in G\} .
$$

Let $K \Subset U$ be an arbitrary compact set, then there is a compact set $L \Subset U, L \supseteq K$ such that $\varphi^{n}(K) \subseteq L$ for every $n \in \mathbb{N}$. Clearly, for every $x \in K$ we have $G_{\rho(x)} \subseteq L$ and, by Proposition 2, $L$ is contained in a hyperbolic open set $V \subseteq U, \varphi(V) \subseteq V$.

The family of sets

$$
G_{\rho(x)}(\varepsilon):=\left\{z \in V: k_{V}\left(z, G_{\rho(x)}\right)<\varepsilon\right\}
$$

is a neighbourhood basis of $G_{\rho(x)}$ and if the orbits falls into $G_{\rho(x)}(\varepsilon)$, it cannot escape because $\varphi$ is a non-expansive with respect to $k_{V}$. For every $\varepsilon>0$ there is a finite set $\left\{x_{1}, \ldots, x_{m}\right\} \subseteq K$ such that

$$
K \subseteq \bigcup_{j=1}^{m} B_{k_{V}}\left(x_{j}, \frac{\varepsilon}{2}\right)
$$

Moreover, there is $n_{0}$ such that for every $n>n_{0}$ and every $j=1, \ldots, m$ we have

$$
k_{V}\left(\varphi^{n}\left(x_{j}\right), G_{\rho\left(x_{j}\right)}\right)<\frac{\varepsilon}{2} .
$$

Clearly, for some $j, G_{\rho(x)} \subseteq G_{\rho\left(x_{j}\right)}\left(\frac{\varepsilon}{2}\right)$. Thus for every $x \in K$ and $n>n_{0}$ for some $j=1, \ldots, m$ we have

$$
\begin{aligned}
k_{V}\left(\varphi^{n}(x), G_{\rho(x)}\right) & <\frac{\varepsilon}{2}+k_{V}\left(\varphi^{n}(x), G_{\rho\left(x_{j}\right)}\right) \\
& \leq \frac{\varepsilon}{2}+k_{V}\left(\varphi^{n}(x), \varphi^{n}\left(x_{j}\right)\right)+k_{V}\left(\varphi^{n}\left(x_{j}\right), G_{\rho\left(x_{j}\right)}\right)<\frac{3}{2} \varepsilon,
\end{aligned}
$$

and orbits of $x \in K$ tend uniformly to $G_{\rho(x)}$.

Since im $P=\operatorname{ker}\left(C_{\varphi}-\right.$ id ) [22, Ch. VIII, Sect. 3] it is easily seen that im $P$ is the set of functions constant on closures of all orbits and for $x \in U$ we have

$$
\overline{O_{x}}=\rho^{-1}(\{\gamma \circ \rho(x): \gamma \in G\}) .
$$

On the other hand, $\operatorname{ker} P=\overline{\operatorname{im}\left(C_{\varphi}-\text { id }\right)}$ [22, Ch. VIII, Sect. 3] and for

$$
S: H(U) \rightarrow H(U), \quad S(f)(z):=\int_{G} f(\gamma \circ \rho(z)) d H(\gamma)
$$

we have ker $S \supseteq \operatorname{im}\left(C_{\varphi}-\right.$ id $)$ and $\operatorname{im} S=\operatorname{im} P$. Therefore $S=P$ on $H(U)$.

Remark 1 . The converse of Theorem 1 is also true. Indeed, for every compact set $K \Subset U$ the set

$$
L_{K}:=\bigcup_{z \in K}\{\gamma \circ \rho(z): \gamma \in G\}
$$


is compact since it is the image of the continuous map

$$
G \times K \rightarrow U, \quad(\gamma, z) \mapsto \gamma(\rho(z)) .
$$

Let $V$ be a relatively compact neighbourhood of $L_{K}$. Since orbits of $z \in K$ tend uniformly to $L_{K}$ there is $n_{0}$ such that $\varphi^{n}(K) \subseteq V$ for every $n>n_{0}$. Thus

$$
\bigcup_{n \in \mathbb{N}} \varphi^{n}(K) \subseteq \bigcup_{n=1}^{n_{0}} \varphi^{n}(K) \cup V
$$

which is relatively compact.

Remark 2. If $U$ is the unit ball then $M$ must be an affine slice of $U$, see [1, Cor. 2.2.16].

The following consequences follow from Theorem 1 in special cases.

Corollary 2 Let $U$ be a connected open subset of $\mathbb{C}$ and let $\varphi: U \rightarrow U$ be a holomorphic self-map. If $C_{\varphi}$ is power bounded then either $\varphi$ is an automorphism of $U$ or all orbits of $\varphi$ tend to a constant $u \in U$ such that $u$ is a fixed point of $\varphi$.

If additionally $U=\mathbb{D}$, then in the automorphism case $\varphi$ has also a fixed point $u$ and

$$
\varphi=\varphi_{u}^{-1} \circ r_{\theta} \circ \varphi_{u}, \quad \theta \in[0,2),
$$

where

$$
\varphi_{u}(z):=\frac{u-z}{1-\bar{u} z}, \quad r_{\theta}(z)=e^{i \theta \pi} z
$$

Moreover, we have

(i) If $\varphi$ is not an automorphism then the projection $P$ associated to $C_{\varphi}$ is given: $P(f)(z)=$ $f(u)$.

(ii) If $\varphi$ is an automorphism and $\theta=\frac{p}{q}$ is rational then $P(f)(z)=\frac{1}{q} \sum_{j=0}^{q-1} f\left(\varphi_{u}^{-1}\left(r_{\frac{j p}{q}}\right.\right.$ $\left.\left.\left(\varphi_{u}(z)\right)\right)\right)$.

(iii) If $\varphi$ is an automorphism and $\theta$ is irrational then $P(f)(z)=\frac{1}{2} \int_{0}^{2} f\left(\varphi_{u}^{-1}\left(r_{\theta}\left(\varphi_{u}(z)\right)\right)\right) d \theta$.

Proof It follows from Theorem 1 in the non-automorphism case (since $M$ has to be zerodimensional and connected). In the automorphism case we apply Denjoy-Wolff theory (see $[19$, Sec. 5]) and the well-known form of automorphisms on $\mathbb{D}$.

Clearly the result above gives a description of mean ergodic composition operators in the case of one-connected one dimensional Riemann manifolds.

Corollary 3 Let $B_{d}$ be the unit ball in a d dimensional complex space $\mathbb{C}^{d}$. Let $\varphi: B_{d} \rightarrow B_{d}$ induce a power bounded map $C_{\varphi}$. Then $\varphi$ satisfies one of the following three properties:

(i) $\varphi$ has a fixed point $u$ and all orbits of $\varphi$ tend to it. In this case $P(f)(z)=f(u)$.

(ii) There is an $m$ dimensional affine submanifold $M$ of $B_{d}, 1 \leq m<d$, (clearly, biholomorphically equivalent to the ball $B_{m}$ in $\mathbb{C}^{m}$ ) with a holomorphic retraction $\rho$ onto $M, \varphi$ restricted to $M$ is an automorphism, it has a fixed point $u$ in $M$ and the projection associated to $C_{\varphi}$ has the form

$$
P(f)=\int_{\Gamma} f\left(\zeta^{-1} \circ A \circ \zeta \circ \rho(z)\right) d H(A),
$$

where $\Gamma$ is a closed subgroup of the group of unitary transformations generated by $\zeta \circ \varphi \circ \zeta^{-1}, H$ is the Haar measure on this group and $\zeta: M \rightarrow B_{m}$ is a biholomorphism such that $\zeta(u)=0$. 
(iii) $\varphi$ is an automorphism of $B_{d}$ with a fixed point and $P$ looks as above for $m=d$.

Proof We apply Theorem 1, and consider separately the cases when $M$ has dimension $0, m$ for $0<m<d$ and $d$. In the second case since $M$ is retractive it must be an affine oneconnected submanifold (see [1, Cor. 2.2.16]), thus it is biholomorphically equivalent to the corresponding $m$ dimensional ball. The rest follows as in the proof of Corollary 2 .

Acknowledgments The authors are very indebted to F. Bracci for providing some information concerning complex dynamics. The second named author is very grateful to colleagues from Valencia for warm hospitality during his stays there.

Open Access This article is distributed under the terms of the Creative Commons Attribution Noncommercial License which permits any noncommercial use, distribution, and reproduction in any medium, provided the original author(s) and source are credited.

\section{References}

1. Abate, M.: Iteration Theory of Holomorphic Maps on Taut Manifolds. Mediterranean Press, Commenda di Rende (1989)

2. Albanese, A.A., Bonet, J., Ricker, W.J.: On mean ergodic operators. In: Curbera, G.P. et al. (eds.) Vector Measures, Integration and Related Topics, vol. 201, pp. 1-20. Operator Theory: Advances and Applications. Birkhäuser Verlag, Basel (2009)

3. Albanese, A.A., Bonet, J., Ricker, W.J.: Mean ergodic operators in Fréchet spaces. Anal. Acad. Sci. Fenn. Math. 34, 401-436 (2009)

4. Bayart, F., Matheron, E.: Dynamics of linear operators. In: Cambridge Tracts in Mathematics, vol. 179. Cambridge University Press, Cambridge (2009)

5. Bernal-González, L., Montes-Rodríguez, A.: Universal functions for composition operators. Complex Var. 27, 47-56 (1995)

6. Bernal-González, L.: Universal entire functions for affine endomorphisms of $\mathbb{C}^{\mathbb{N}}$. J. Math. Anal. Appl. 305, 690-697 (2005)

7. Bonet, J., Domański, P.: Power bounded composition operators on spaces of analytic functions. Collect. Math. doi:10.1007/s13348-010-0005-9 (2010)

8. Cowen, C.C., MacCluer, B.D.: Composition operators on spaces of analytic functions. In: Studies in Advanced Mathematics. CRC Press, Boca Raton (1995)

9. Fonf, V.P., Lin, M., Wojtaszczyk, P.: Ergodic characterizations of reflexivity in Banach spaces. J. Funct. Anal. 187, 146-162 (2001)

10. Grosse-Erdmann, K.G.: Universal families and hypercyclic operators. Bull. Am. Math. Soc. 36, 345-381 (2001)

11. Grosse-Erdmann, K.G.: Recent developments in hypercyclicity. Rev. R. Acad. Cien. Ser. A Math. 97, 273-286 (2003)

12. Grosse-Erdmann, K.G., Mortini, R.: Universal functions for composition operators with non-automorphic symbol. J. Anal. Math. 107, 355-376 (2009)

13. Grosse-Erdmann, K.G, Peris, A.: Linear Chaos. Springer, Berlin (2011)

14. Gunning, R.C.: Introduction to Holomorphic Functions of Several Variables. Wadsworth \& Brooks, Belmont (1990)

15. Hörmander, L.: An Introduction to Complex Analysis in Several Variables, 3rd edn. North-Holland, Amsterdam (1990)

16. Kobayashi, S.: Hyperbolic Complex Spaces. Springer, Berlin (1998)

17. Krengel, U.: Ergodic Theorems. de Gruyter, Berlin (1985)

18. Meise, R., Vogt, D.: Introduction to Functional Analysis. Clarendon Press, Oxford (1997)

19. Milnor, J.: Dynamics in One Complex Variable. Vieweg Verlag, Wiesbaden (2000)

20. Shapiro, J.H.: Composition operators and classical function theory. In: Universitext: Tracts in Mathematics. Springer, New York (1993)

21. Shapiro, J.H.: Notes on the Dynamics of Linear Operators. Lecture notes. http://www.math.msu.edu/ $\sim$ shapiro/Pubvit/Downloads/LinDynamics/lindynamics.pdf (1993)

22. Yosida, K.: Functional Analysis. Springer, Berlin (2001) 